\title{
Prontuário Psicológico Orientado para o Problema: Um Modelo em Construção
}

Problem-oriented psychological record: a model in construction

Fabrício Fernandes Almeida Clara Cantal Áderson Luiz Costa Junior Universidade de Brasília
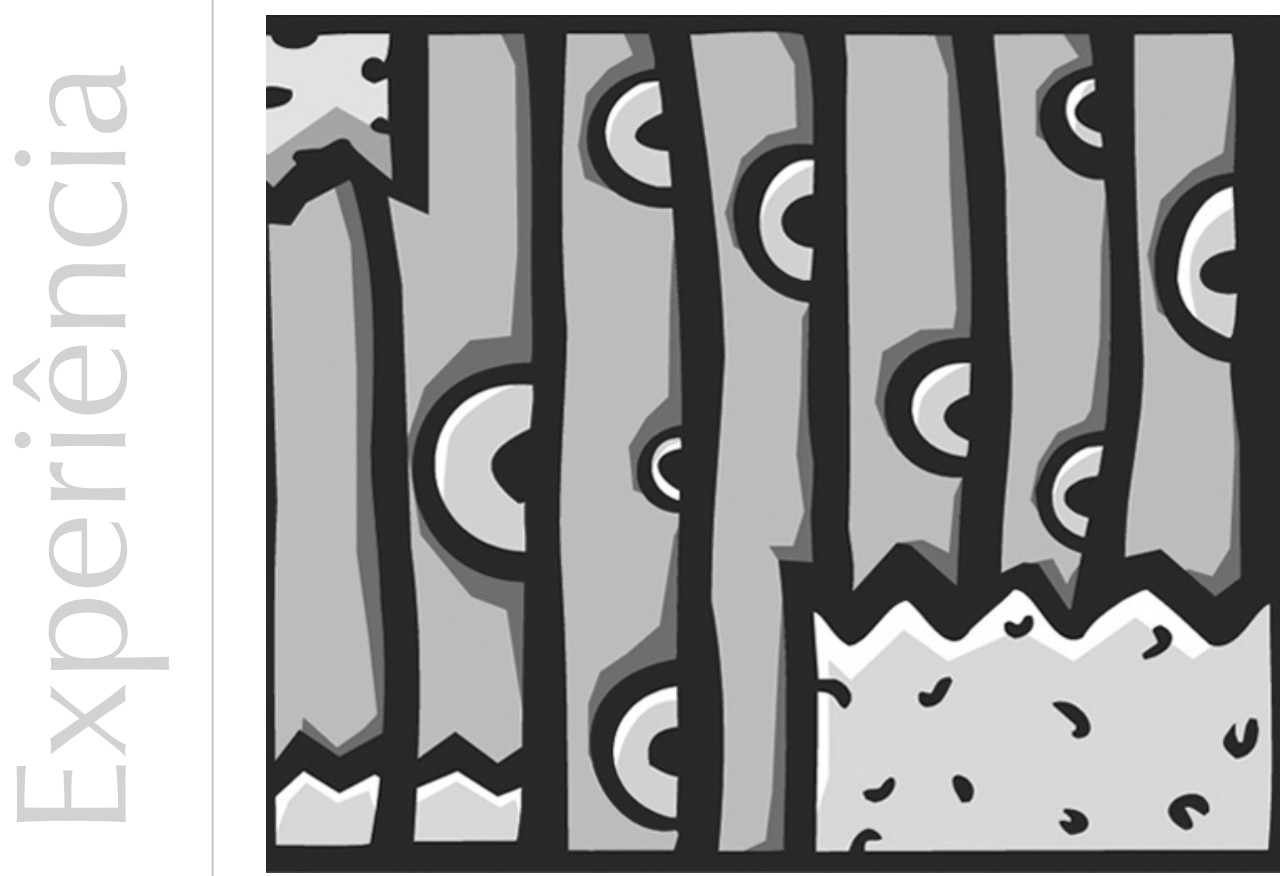
Resumo: Este artigo tem como objetivo apresentar uma proposta de modelo de anotações psicológicas em prontuário que possibilite o compartilhamento de informações relacionadas ao paciente em um contexto hospitalar, respeitando a confidencialidade requerida do profissional de Psicologia, tornando essas informações pertinentes e acessíveis a toda a equipe de saúde e adaptando-as às demandas de uma equipe transdisciplinar. A confecção do modelo de anotação psicológica em prontuários foi realizada a partir da adaptação de modelos adotados pela ciência médica e subdividida em três etapas: realização de revisão bibliográfica, confecção do modelo de anotações psicológicas e testagem prática dos instrumentos. A versão final do modelo de prontuário psicológico orientado para o problema (PPOP) consiste do formulário de atendimento inicial do PPOP e dos formulários de notas de evolução psicológica do PPOP. Esse modelo encoraja uma abordagem lógica ao diagnóstico e ao tratamento e prioriza a ordem prática do cuidado transdisciplinar.

Palavras- chave: Prontuário psicológico. Sistemas de registro. Psicologia da saúde. Hospital.

\begin{abstract}
The objective of this article is the presentation of a proposal for a model of psychological documentation in patient records that makes possible the sharing of information related to the patient in a hospital context, regarding the confidentiality that is required of the Psychology professional, making this information accessible and pertinent to the entire health team and adapting this information to the demands of a transdisciplinar health team. The confection of the psychological documentation in the patient records model went through the adaptation of the models adopted by the medical science and was subdivided in three steps: the study of bibliographical review, the elaboration of the psychological documentation model and the instruments' practical trial. The final version of the proposal for the problem-oriented psychological record model (PPOP) consists of the PPOP form for initial care and the PPOP forms of psychological evolution documentation. This model encourages a logical approach to diagnosis and treatment, giving priority to the transdisciplinary care practical order.
\end{abstract}

Keywords: Psychological record. Registration systems. Health Psychology. Hospital.

Por muitos anos, a oferta de serviços voltados para a saúde mental tem sido o principal foco da prática profissional da Psicologia no Brasil. Contudo, nas últimas décadas do século XX, com a criação da Psicologia da saúde enquanto área de conhecimento da American Psychological Association e a inclusão da Psicologia como área da saúde pelo Conselho Nacional de Saúde do Ministério da Saúde, as demandas e as atribuições da Psicologia, no contexto de tratamentos de saúde, tornaram-se muito mais amplas que aquelas relacionadas à avaliação e à intervenção em saúde mental de indivíduos e grupos. Essa nova configuração é, em grande parte, resultado do próprio processo de crescimento da Psicologia da saúde, que inclui a inserção crescente de psicólogos em instituições hospitalares como membros de equipes de diferentes áreas de especialidades da Medicina, enfermagem, Odontologia e outras áreas da saúde (Belar, 1997; Costa Junior, 2005).

A atuação da Psicologia na área da saúde surge no início do século XX como proposta legítima de conhecimento, a partir da necessidade 
A Psicologia da saúde, como área de conhecimento, representa uma proposta teóricometodológica que prioriza a promoção de repertórios de comportamento voltados para a saúde enquanto fenômeno social, como forma de garantir a continuidade do processo de desenvolvimento humano, individual e coletivo (Matarazzo, 1980). de saberes mais específicos de áreas como a Psicologia clínica, a Medicina psicossomática e a Psicologia social comunitária, e da proposta de integrar a Psicologia à educação médica formal. Dessa forma, o modelo adotado foi o médico-biológico, segundo uma visão cartesiana. Para tanto, a atuação esteve mais voltada para a humanização dos atendimentos aos pacientes e para a compreensão de padrões de personalidade relacionados à saúde (Gioia-Martins \& Rocha, 2001).

Segundo Sebastiani (2003), os marcos de inicialização da Psicologia da saúde no Brasil datam da década de 50, e são anteriores, portanto, à própria regulamentação da profissão de psicólogo neste país, datada de 1961. Ao final da década de 70, iniciamse as produções científicas e as primeiras experiências de formação acadêmica nessa área, e ocorrem diversos concursos públicos em instituições municipais, estaduais e federais de saúde para a contratação de psicólogos com o objetivo de desenvolver atividades profissionais e pesquisa científica em hospitais, ambulatórios, unidades básicas de saúde, centros de saúde e programas de orientação, prevenção e educação para a saúde.

Nessa mesma década, Matarazzo (1980) caracteriza a Psicologia da saúde, conforme preconizado pela Divisão 38 da American Psychological Association, como a área de conhecimento e de atuação da Psicologia que se ocupa da promoção e da manutenção da saúde e da prevenção e tratamento de doenças, interessando-se pela investigação de variáveis psicossocias relacionadas ao desenvolvimento e à reabilitação de processos patológicos em contextos de tratamento médico, odontológico e outros. Trata-se de uma abordagem educativa que prioriza o desenvolvimento de comportamentos voltados para a saúde de indivíduos e grupos, destacando-se medidas de melhoria da adesão a tratamento e de estratégias de enfrentamento de tratamentos de saúde e doenças físicas, agudas e crônicas. Já a Psicologia hospitalar, segundo Costa Junior (2005), constitui uma vertente da Psicologia da saúde cuja atuação se restringe aos contextos de unidades hospitalares e inclui um conjunto de intervenções profissionais da Psicologia com o objetivo de minimizar o sofrimento percebido, ou provocado, pela experiência de hospitalização (doença, procedimentos médicos invasivos, seqüelas e decorrências emocionais da internação).

\section{A atuação em psicologia da saúde e o registro em prontuários médico- hospitalares}

A Psicologia da saúde, como área de conhecimento, representa uma proposta teórico-metodológica que prioriza a promoção de repertórios de comportamento voltados para a saúde enquanto fenômeno social, como forma de garantir a continuidade do processo de desenvolvimento humano, individual e coletivo (Matarazzo, 1980).

Seu objeto de estudo, o processo saúdedoença, entendido como um fenômeno coletivo, num processo histórico e multideterminado, exige uma atuação integrada com vistas à saúde, demonstrando a necessidade do desenvolvimento de atividades interdisciplinares entre os profissionais de saúde. Assim, o movimento da saúde integral, a visão biopsicossocial, influencia uma nova forma de atuação e enfatiza a melhoria de qualidade de vida no trabalho e o direito 
que todo cidadão tem de receber atenção e cuidados que garantam o atendimento global às suas necessidades. O psicólogo da saúde se ocupa, então, da necessidade de promover e de considerar o processo saúde-doença como um fenômeno social. Além disso, os crescentes custos dos serviços de saúde têm colocado em evidência a importância da educação sobre práticas saudáveis e políticas de prevenção que permitam uma intervenção global, o aumento dos índices de adesão a tratamentos e a redução do impacto da doença sobre o funcionamento global do indivíduo (Gioia-Martins \& Rocha, 2001).

Nesse contexto de atenção e intervenção globais à saúde, que se faz presente no modelo biopsicossocial ao qual a Psicologia da saúde vem associar-se, fazem-se necessárias formas de atender as diversas demandas do paciente internado em uma instituição hospitalar, sem priorizar necessariamente as problemáticas físicas e biológicas e sem perder de vista a intervenção multi e interdisciplinar. As informações colhidas pelos profissionais de Psicologia devem fazer parte do prontuário desses pacientes, de forma a adicionar uma dimensão mais funcional e humana ao "ente" chamado de "paciente".

No entanto, a disponibilidade de estudos brasileiros que apontem diretrizes ou fomentem uma discussão acerca da forma como essas informações devem ser divididas entre os psicólogos da saúde e outros profissionais das equipes de saúde é extremamente rara, o que tem gerado grandes discrepâncias entre o modo de atuar das diferentes equipes de saúde até mesmo dentro da mesma área de especialidade, o que dificulta a padronização de um modelo comum de registro.

Podemos afirmar que a forma mais segura de registro e divisão de informação em uma instituição de saúde é realizada por meio do prontuário do paciente ou, dentre diversos outros adjetivos, do ainda denominado prontuário médico. Seja qual for o adjetivo que o acompanhe, o prontuário é um produto de diversos estudos que procuraram aprimorar seu formato, tais como o de Kluck e Guimarães (2002), o de Lopes (1999), o de Massad, Marin e Azevedo Neto (2003) e o de Nascimento, Andrade, Assed e Brasil (2006). Hoje o prontuário está presente no dia a dia de todos os profissionais que atuam em um hospital, unidade ambulatorial, posto de saúde, etc. Contudo, o registro do psicólogo no prontuário ainda não é uma rotina em toda instituição de saúde; observa-se uma carência de divulgação de modelos de anotação em Psicologia e, especialmente, a ausência de um modelo padronizado de anotações, o que poderia facilitar a implementação de uma forma eficaz de registros, organizados temporal e funcionalmente, cujas informações dispersas estivessem inter-relacionadas para posterior recuperação, análise e interpretação, por meio de um denominador comum: o problema de saúde que motivou o tratamento. Além disso, ainda se observa uma predominância de modelos médico-biológicos de atuação em saúde, que se caracterizam por uma filosofia de ação determinada por ações médicas voltadas para os elementos prioritariamente biológicos do tratamento.

Weed (1969, cf. Lopes, 1999) propôs um modelo de prontuário médico orientado por problemas (POPE). Segundo o autor, esse tipo de prontuário é um modelo amplamente aceito para padronizar e melhorar a qualidade do conteúdo dos prontuários médicos. Tal modelo frisa a necessidade de se obter todas as informações, dados socio-demográficos, dados pessoais, sintomas, sinais, testes e exames específicos, a fim de construir uma hierarquia de problemas. 
Dentro dessa lista de problemas, o autor diferencia-os em ativos ou inativos. Os problemas ativos dizem respeito às questões que necessitam de atenção contínua dos profissionais de saúde envolvidos com os cuidados ao paciente ou que causam algum tipo de desconforto ao paciente no momento da internação; os problemas inativos dizem respeito a questões já solucionadas ou que, apesar do risco de recidiva ou complicação, não provocam incômodo para o paciente e não requerem vigilância contínua durante a internação corrente, embora demandem avaliações periódicas.

Dentro do prontuário orientado por problemas (POPE), Weed (1969, cf. Lopes, 1999) ainda propõe um modelo de anotações em prontuário. $\mathrm{O}$ autor pontua que essas notas de evolução são orientadas pelos problemas ativos do paciente e seguem o contexto descrito como S.O.A.P. Nessa concepção, cada uma das letras do S.O.A.P. se refere aos quatro aspectos considerados fundamentais nas notas de evolução diária. Segundo Massad et al. (2003), esses fatores são as queixas do paciente - subjetividade do paciente (S), as verificações do médico - o que é observado pelo profissional (O), os testes e as conclusões a respeito do caso - avaliação do profissional (A) e o plano de cuidado - prognóstico do paciente (P).

Três décadas mais tarde, Nascimento et al. (2006) propõem um modelo de anotação psiquiátrica em prontuário que deve incluir cabeçalho, motivo do pedido, anamnese, dados de exame psíquico e exame físico do paciente, diagnóstico e recomendações. Segundo os autores, no cabeçalho deve constar registro do atendimento (avaliação inicial, primeira entrevista, segunda entrevista, e assim por diante) com suas respectivas datas e horários, além das fontes de informação utilizadas (entrevista do paciente ou de familiares, revisão de prontuário, resultados de exames). Já o motivo do pedido deve explicitar quem solicitou o parecer, abrigando trechos transcritos do pedido de parecer ou tradução sucinta das demandas do parecer, direcionando assim o foco do atendimento e facilitando o planejamento das ações junto ao paciente.

Na anamnese, devem constar as informações do histórico pessoal de vida do paciente. Os autores sugerem que esse item seja subdividido em identificação, queixa principal e histórico da doença, dados da história fisiológica, patológica pregressa, familiar e pessoal, a fim de reduzir a possibilidade do esquecimento do registro de informações consideradas relevantes. Os autores destacam, ainda, o cuidado que deve ser tomado com as normas de sigilo profissional de cada categoria bem como com a utilização de linguagem adequada ao entendimento dos outros profissionais que compõem a equipe.

Nesse modelo, o exame psíquico se refere ao registro das funções psíquicas básicas, tais como aparência, atitude, consciência, orientação, atenção, memória, inteligência, senso-percepção, fala, atenção, humor, afeto, consciência do eu, vontade, pragmatismo, consciência da morbidade e planos para o futuro. Nessa concepção, o exame físico abrange os aspectos estruturais (físicos, biológicos) observados pelo psiquiatra que podem ser importantes para a diferenciação de transtornos psiquiátricos. Segundo os autores, o diagnóstico deve ser estruturado em linguagem médica, de forma padronizada e clara para as diversas especialidades que compõem a equipe de saúde, enquanto a seção de recomendações deve incluir as anotações necessárias ao esclarecimento do diagnóstico e às prescrições. 
O estudo de Azevedo e Thomas (2002) fornece algumas diretrizes para a construção do registro psicológico em prontuário, apesar de não delimitar o formato do instrumento. O trabalho informa e expõe a necessidade de se formular um modelo de anotações em prontuário para o serviço de Psicologia em hospitais e instituições de saúde, de modo a facilitar o entendimento do quadro do paciente.

Massad et al. (2003) observam que a Organização Pan-Americana de Saúde (OPAS) e a Organização Mundial da Saúde (OMS) têm estimulado a confecção de prontuários eletrônicos do paciente (PEP), forma proposta para unir diferentes tipos de dados produzidos em variados formatos, em épocas diferentes, elaborados por diferentes profissionais da equipe de saúde e em locais distintos.

\section{O amplo espectro de situações problemáticas em saúde, naquelas em que o psicólogo deve enfrentar para responder as demandas existentes, o obrigam a fazer uso dos recursos teórico- metodológicos disponíveis assim como implementar intervenções que nem sempre são possíveis de se conjugar com um enquadre teórico da Psicologia. ...}

(p. 30)
Segundo Sebastiani (2003), a Associação Latino-americana de Psicologia da Saúde estabeleceu, em 1997, que:

\section{O amplo espectro de situações problemáticas em saúde, naquelas em que o psicólogo deve enfrentar para responder as demandas existentes, o obrigam a fazer uso dos recursos teórico-metodológicos disponíveis assim como implementar intervenções que nem sempre são possíveis de se conjugar com um enquadre teórico da Psicologia. ... (p. 30)}

Dessa forma, este trabalho tem como objetivo apresentar uma proposta de modelo de anotações psicológicas em prontuário por meio da adaptação de modelos adotados pela área médica. Mais especificamente, o texto descreve um modelo que possibilita o compartilhamento das informações de acompanhamento dos pacientes, respeitando a confidencialidade das informações privilegiadas, tornando as informações pertinentes acessíveis a toda a equipe e permitindo a transformação da própria equipe de multiprofissional a transdisciplinar (os diversos profissionais da equipe trocam informações e discutem as melhores soluções para o paciente, atuando conjuntamente). Cabe pontuar que o modelo foi gerado e adaptado para as demandas do Serviço de Psicologia da Clínica Cirúrgica do Hospital Universitário de Brasília (HUB).

\section{Metodologia}

A confecção do modelo de anotação psicológica em prontuários foi subdividida em três etapas: (a) realização de revisão bibliográfica em busca de modelos de anotação em prontuários já consolidados, (b) confecção do modelo de anotações psicológicas e (c) testagem prática do instrumento.

\section{Revisão bibliográfica}

Efetuou-se contato com o Conselho Regional de Psicologia - 1a Região (CRP-01) com o objetivo de obter informações a respeito de normas técnicas sobre anotações de Psicologia em prontuários. Diante da informação de inexistência de tais normas por parte do CRP-01, realizou-se um levantamento bibliográfico sobre a legislação pertinente à confecção de prontuários nas diversas áreas de cuidados com a saúde. O levantamento identificou, entre outros itens, a publicação da OPAS/OMS, que direcionou as normas de anotação em prontuário adotadas pelo modelo proposto por Weed (1969). Foram ainda identificados três trabalhos relacionados ao modelo de Weed (1969) e trabalhos adicionais relacionados à elaboração de prontuários, objetos de discussão ao longo deste estudo. 


\section{Confecção do modelo de anotações psicológicas em prontuário}

Finalizada a etapa de revisão bibliográfica, iniciou-se a elaboração do modelo de anotações psicológicas a partir da adaptação do modelo POPE (Weed, 1969) às demandas do Serviço de Psicologia da Clínica Cirúrgica do HUB.

Inicialmente, estagiários de Psicologia e a psicóloga da unidade deliberaram a respeito dos dados de interesse a serem coletados em entrevista inicial, considerando-se a realidade da unidade e suas demandas em um contexto transdisciplinar de atenção ao paciente. A partir de uma versão preliminar de entrevista inicial, elaborada por Turra (2006) e já utilizada nessa unidade, elaborou-se o instrumento de coleta de dados denominado "atendimento inicial PPOP".

Essa evolução inicial, segundo o modelo POPE (Weed, 1969), descreve uma listagem de problemas ativos e inativos do paciente, conforme as quatro facetas preconizadas pelo SOAP, bem como a necessidade e o grau de prioridade no atendimento do paciente consultado. A padronização dos dados foi resultado da demanda específica do serviço de Psicologia da unidade e das demandas legais e éticas envolvidas no exercício da profissão de psicólogo em contexto hospitalar.

Respeitando a lista de prioridades diária, constaram ainda no prontuário anotações de visitas posteriores que relatavam a evolução do atendimento diário ao paciente. Tais anotações de evolução psicológica foram concebidas em duas vias: uma para o prontuário psicológico denominada "notas de evolução psicológica", e que era arquivada pelo serviço de Psicologia, e outra para o prontuário do paciente, denominada "evolução psicológica - notas de prontuário", de acesso irrestrito aos outros profissionais de saúde envolvidos nos cuidados com o paciente durante seu tratamento. O procedimento objetivou cumprir o art. 9o do Código de Ética do Profissional Psicólogo (Conselho Federal de Psicologia [CFP], 2005) que estabelece que "é dever do psicólogo respeitar o sigilo profissional a fim de proteger, por meio da confidencialidade, a intimidade das pessoas, grupos ou organizações, a que tenha acesso no exercício profissional" (p. 13).

Ao fim da internação do paciente na Clínica Cirúrgica do Hospital e conseqüente alta pelo serviço de Psicologia, as notas de evolução psicológica são arquivadas em local próprio e de acesso restrito, sob os cuidados privativos da Divisão de Psicologia do HUB.

\section{Testagem prática do instrumento}

Após a confecção do instrumento inicial, realizou-se uma testagem prática entre os meses de outubro e dezembro de 2006, o que possibilitou uma ampla revisão do instrumento, incluindo a resolução de dúvidas e a identificação de necessidades e ambigüidades. Após três versões, foi produzida a versão final do instrumento.

\section{Resultados e discussão}

O modelo de prontuário psicológico orientado para o problema (PPOP), cuja versão final 
consiste do formulário de atendimento inicial do PPOP e dos formulários de notas de evolução, inclui :

\section{Protocolo de atendimento inicial do PPOP}

No protocolo de atendimento inicial do PPOP, podem ser discriminados: cabeçalho, descrição de rede de apoio, local e qualidade de residência, descritores empregatícios e de benefícios sociais, lista de problemas ativos e inativos, facilidades e dificuldades enfrentadas durante a internação no serviço, descritores da dor atual (caso haja), descritor de compreensão sobre o quadro de saúde e situação cirúrgica e, por fim, uma versão inicial do S.O.A.P.

\section{Cabeçalho}

No cabeçalho, devem ser descritos o dia de realização da anotação, a clínica, a especialidade do tratamento, o crondalfa (código que remete ao paciente e que não muda como o prontuário), o número do prontuário, o nome do paciente, a enfermaria de internação, a idade do paciente, o nome e o vínculo do cuidador e um número de telefone para contato. Tais informações possibilitam a identificação do paciente e a posterior localização do prontuário por diferentes profissionais. Ao contrário do que apontam Massad et al. (2003), a maior parte dos prontuários hospitalares ainda não está disponível em um sistema capaz de ser acessado por diversos profissionais de diferentes serviços de saúde, mas está disponível apenas para os profissionais de saúde do hospital onde a internação e/ou o tratamento ocorreram.

\section{Descrição de rede de apoio}

O instrumento também reserva espaço para anotações concernentes à rede de suporte social do paciente. Essa opção se justifica devido à relevância de se avaliar a possibilidade de transposição entre problemas ativos e inativos, além de possibilitar o planejamento de preparação para a alta hospitalar.

Segundo Thoits (1995, cf. Seidl \& Tróccoli, 2006), os estudos em suporte social podem ser divididos em duas categorias designadas de acordo com a conceituação e o tipo de enfoque utilizados: (a) estrutural e (b) funcional. Segundo o autor, a abordagem estrutural registra a freqüência e a quantidade das relações sociais do indivíduo, enquanto a abordagem funcional refere-se à extensão em que as relações sociais podem cumprir determinadas funções, voltando-se para a percepção quanto à disponibilidade e o tipo de apoio recebido e abarcando ainda a satisfação com o mesmo.

Para Seidl e Tróccoli (2006), dois constituintes da abordagem funcional têm prevalecido na literatura: o suporte operacional ou instrumental e o suporte emocional ou de estima. O suporte instrumental ou operacional remete à disponibilização de ajuda que auxilie a pessoa no manejo ou resolução de situações práticas ou operacionais do cotidiano, como apoio material, financeiro ou das atividades diversas do dia a dia. O suporte emocional ou de estima remete a comportamentos, como escutar, prover atenção ou fazer companhia, que contribuam para que a pessoa se sinta cuidada e/ ou estimada.

A exemplo dos autores citados, optou-se pelo enfoque funcional do fenômeno no 
instrumento. Considerando que o juízo do paciente a respeito do suporte social recebido constitui um dos indicadores de julgamento de efetividade da rede de apoio, estruturouse uma lista com três tópicos que servem de guia para a entrevista de natureza semiestruturada:

(1) Quem é o provedor da casa onde mora? Alguém divide as despesas com você? Quão satisfeito está com isso? (instrumental financeiro);

(2) Quando não está se sentindo bem, existe alguém que o ajude a limpar a casa, que cozinhe para você ou que até mesmo o leve ao hospital e o acompanhe no mesmo? Quão satisfeito está com isso? (instrumental - material);

(3) Existe alguém com quem possa desabafar, conversar e que faça você se "sentir bem"? Quão satisfeito está com isso? (emocional).

\section{Local e qualidade de residência}

Este tópico destina-se à apresentação do contexto instrumental e funcional de moradia do paciente. Esses dados são relevantes para a previsão de situações que necessitem de algum tipo de manejo durante a internação e que apresentem potencial de alteração de status, podendo migrar de problemas inativos para problemas ativos, como pacientes que residem fora da cidade onde ocorre o atendimento, por exemplo, e que têm familiares hospedados em hotéis e que, por motivos clínicos, são obrigados a estender a internação hospitalar por períodos superiores ao previstos inicialmente.
Tais dados, associados às informações de descrição de rede de apoio, ajudam também na percepção da necessidade de um trabalho mais individualizado ou de cunho mais familiar no momento que permeia a alta. Dessa forma, o atendimento psicológico e da equipe transcendem as paredes da instituição hospitalar e chegam ao ambiente doméstico das pessoas atendidas pelo serviço.

\section{Descritores empregatícios e de benefícios sociais}

Os descritores empregatícios e de benefícios sociais existentes nesse protocolo são condizentes com o tipo de atendimento proposto por esse modelo, orientado para o problema e que prevê uma equipe transdisciplinar. Tais dados, associados aos de rede de apoio e moradia, auxiliam a identificação de necessidades de parecer técnico por parte de profissionais de serviço social, eliminando a necessidade de uma nova avaliação ou mesmo de uma entrevista inicial do(a) assistente social com o paciente e permitindo que o(a) mesmo(a) colete apenas os dados de interesse exclusivo de sua profissão ou os que sejam relevantes ao caso em atendimento.

\section{Lista de problemas ativos e inativos}

Na lista de problemas ativos, devem aparecer as questões que afligem o paciente durante o período de internação e/ou tratamento. A lista de problemas ativos não deve descrever problemas que, apesar de existirem, não são sentidos ou mencionados pelo paciente como empecilhos no momento da internação, ou seja, são problemas inativos (Lopes, 2005). 


\section{Facilidades e dificuldades enfrentadas durante a internação}

As facilidades e dificuldades enfrentadas exprimem as estratégias de coping adotadas pelo paciente durante a sua internação e/ou tratamento atual. Segundo Lazarus e Folkman (1986, cf. Poch \& Caparrós, 2000), o coping pode ser caracterizado como as exigências internas e/ou externas geradas para dominar, reduzir ou tolerar as exigências internas e/ou externas criadas pela exposição a situações estressantes. Na área da saúde, os estudos de coping se interessam prioritariamente em investigar como pacientes e acompanhantes lidam com os eventos estressantes que envolvem o tratamento de saúde e como intervenções psicológicas podem facilitar o desenvolvimento de estratégias mais eficientes de enfrentamento de doenças e processos de tratamento/hospitalização (Costa Junior, 2005).

Assim, investigando-se a forma como os pacientes reagem à internação e/ou tratamento, é possível traçar planos de cuidados individualizados e condizentes com a percepção e com o repertório comportamental do paciente no hospital. Além disso, os planos e estratégias de cuidados com o paciente podem ser traçados mais objetivamente e de forma mais pontual, lidando com os problemas ativos de maneira mais eficiente e eficaz. Em conseqüência, optou-se por campos de anotação textual para a livre expressão dos pacientes respondentes.

\section{Descritores da dor atual}

O significado idiossincrásico atribuído à dor é o resultado de uma série de pontos articulados entre essas variáveis e características que se interseccionam. As atitudes explícitas, observáveis, e os comportamentos manifestos são o que o observador pode ver, compreender e, na maior parte das vezes, inferir sobre o que observa (International Association for the Study of Pain Press [IASP], 1995).

Dessa forma, é importante que os dados colhidos durante a entrevista inicial e durante as avaliações diárias sejam os mais próximos da realidade do paciente, sendo ele o único capaz de descrever sua experiência de dor e o significado da mesma. Em conseqüência, optou-se, como nos campos avaliativos do coping, por uma metodologia de anotação textual direta e fiel aos descritores citados pelo paciente.

Os elementos relevantes nos cuidados com o paciente que experimenta dor dizem respeito à sua localização, temporalidade, intensidade, qualidade e fatores de alívio e exacerbação. Assim, no momento da avaliação dos dados colhidos nesse campo, deve-se optar pela ordenação dos descritores na ordem citada de seus elementos relevantes, observando-se o diagrama de localização da dor contido no protocolo, a evolução da dor durante o tempo de internação - no caso particular da clínica cirúrgica, observá-la no pré, trans e pósoperatório -, a variação no valor da escala de intensidade da dor, a alteração dos descritores qualitativos da dor e, por fim, a presença ou ausência de fatores funcionais relacionados ao alívio ou à exacerbação.

\section{Descritor de compreensão sobre o quadro de saúde atual}

Dentre as diversas atividades exercidas pelo profissional de Psicologia da saúde, está a 
mediação da comunicação equipe-paciente e vice-versa.

Segundo Fabbro (1999), a Resolução do Conselho Federal de Medicina no 1.246/88, art. 46, reafirma o papel do consentimento informado do paciente. As informações prestadas pelo médico referentes às condições clínicas do paciente sobre as alternativas terapêuticas, com suas chances de sucesso, riscos, possibilidade de seqüelas ou de complicações que poderão ocorrer, ajustadas às possibilidades de compreensão do paciente ou de seu representante legal, são pressupostos do consentimento esclarecido, quer para a realização dos procedimentos necessários à formulação do diagnóstico, quer para a realização da terapêutica indicada.

Dessa forma, julgou-se necessário abordar a compreensão do paciente sobre seu quadro de saúde. Ressalta-se a importância de o paciente compreender sua condição de saúde, uma vez que tal entendimento constitui um dos indicadores de adesão ao tratamento e de seguimento das prescrições terapêuticas após a internação (Arora, 2003).

\section{Nível atual de satisfação do paciente e cuidador com o tratamento}

O nível atual de satisfação do paciente e do cuidador com o tratamento foi mensurado por meio de uma escala Likert de 10 pontos, na qual o número um representa insatisfação total com o tratamento e o número 10 representa satisfação total com o tratamento. Esse dado foi colhido com o objetivo de avaliar, de forma geral, o impacto dos construtos inferidos pelo atendimento inicial do PPOP - coping, rede de suporte social, dor, etc - na experiência de internação do paciente e de seu acompanhante.

\section{Versão inicial do S.O.A.P.}

Na ficha também pode ser observada a presença do S.O.A.P. inicial. Contudo, as anotações pertencentes a essa etapa da evolução sofrem a influência direta do serviço ao qual pertencem. Como apontado por Weed (1969, cf. Lopes, 1999), uma anotação completa deve conter: os apontamentos do paciente relativos a sua subjetividade e história de vida (S), os aspectos observados durante o atendimento $(\mathrm{O})$, tais como aparência, atitude, consciência, orientação, atenção, memória, inteligência, senso-percepção, fala, atenção, humor, afeto, consciência do eu, vontade, pragmatismo, consciência da morbidade e prospecção - todos pertencentes à classe das funções psíquicas -, avaliação a respeito do estado do paciente e diagnóstico do paciente, se esse for o caso (A), e prognóstico do paciente, com delimitação do atendimento prestado e condutas futuras para a equipe (P).

\section{Protocolos de notas de evolução do PPOP}

Nos protocolos de notas de evolução do PPOP, podem ser discriminados os mesmos campos de cabeçalho, lista de problemas ativos e do S.O.A.P. presentes no protocolo de atendimento inicial. Contudo, tais campos apresentam pequenas alterações que são condizentes com sua situação de anotações diárias.

Ao campo de cabeçalho, adiciona-se o número de dias de internação do paciente no serviço 
e o motivo do atendimento. Tais informações são relevantes para o acompanhamento da evolução dos planos terapêuticos traçados no campo (P) do S.O.A.P., além de remeter às possibilidades de atendimento prioritário, por ronda ou solicitação de parecer. No caso de solicitação por parecer, deve ser explicitado o serviço solicitante (Medicina, enfermagem, serviço social, outros).

No que diz respeito à lista de problemas ativos e ao S.O.A.P. contido no formulário, ambos requerem atualização a cada consulta, resultando em um plano terapêutico que permite a continuidade de condutas previamente traçadas ou a elaboração de novas diretrizes e condutas de atendimento ao paciente.

Apesar do modelo de prontuário psicológico orientado para o problema apresentar duas vias de notas de evolução, não há distinção quanto à estruturação desses instrumentos - salvo o título do mesmo -, cabendo ao avaliador a obrigatoriedade de cumprir o art. 9o do Código de Ética do Profissional Psicólogo (CFP, 2005). Dessa forma, cada evolução pode ou não gerar dois protocolos de notas de evolução do PPOP diferentes.

\section{Considerações finais}

As notas psicológicas em prontuário podem constituir uma das formas de firmar a presença e a necessidade do psicólogo como membro efetivo de equipes de saúde que atuam em unidades hospitalares, além de priorizar o processo de atenção integral à saúde e servir de fonte de informação e pesquisa educacional, clínica e administrativa para a tomada de decisões sobre o tratamento e as necessidades do paciente. Além disso, deve servir de meio de comunicação compartilhado entre todos os profissionais de saúde, aproximando a equipe de um contexto de trabalho transdisciplinar.

Entretanto, para serem cumpridas essas funções, é preciso que as notas psicológicas sejam redigidas de modo claro e acurado; para tanto, deve ser dado treinamento aos profissionais psicólogos pelas instituições, a fim de facilitar a utilização e a escrita no formato proposto, o que proporcionará uma comunicação transdisciplinar efetiva.

A padronização de um modelo de prontuário psicológico orientado para o problema, a divisão desse modelo em seções e o estabelecimento de itens a serem respondidos e observados em cada seção facilitam a elaboração do prontuário e evitam falhas e omissões no preenchimento do mesmo, tornando-o um instrumento mais confiável e eficiente e facilitando a compreensão pelos diversos profissionais que o manuseiam.

Esse modelo encoraja, ainda, uma abordagem lógica para o diagnóstico e o tratamento, priorizando a questão de se manter em ordem a prática do cuidado transdisciplinar.

É importante ressaltar o cuidado que deve ser tomado em expor apenas o necessário, as informações que o profissional de Psicologia julga relevantes no quadro do paciente ou como resposta a parecer(es) solicitado(s), nas notas de evolução psicológica adicionadas ao prontuário do paciente. Essa é uma medida importante no resguardo da confidencialidade do paciente e que é preconizada pelo Código de Ética do Profissional Psicólogo. Julgamos que, ao utilizar o modelo proposto, o profissional psicólogo deve exercitar os princípios éticos da profissão, considerando 
o que é importante relatar no prontuário do paciente e o que é necessário relatar apenas nas notas de evolução psicológica restritas à utilização do serviço de Psicologia.

Com a utilização prática do modelo, reparamos que, por vezes, o trecho relativo ao $\mathrm{S}$. do modelo fica mais curto nas notas de evolução psicológica voltadas para o prontuário do paciente do que naquelas voltadas e restritas ao serviço de Psicologia. Isso implica menor exposição do relato do paciente, mantendo em sigilo as informações pessoais fornecidas por ele ou pelo seu acompanhante que não tenham qualquer função na resolução do caso ou da resposta a um parecer.

Destaca-se que mais estudos são necessários para avaliar a utilização e a adaptação desse modelo a contextos específicos de atuação de psicólogos da saúde em instituições hospitalares. 


\section{Fabrício Fernandes Almeida *}

Psicólogo graduado pela Universidade de Brasília e membro da equipe de Psicologia do Hospital Universitário de Brasília.

\section{Clara Cantal}

Estudante de graduação em Psicologia da Universidade de Brasília

\section{Áderson Luiz Costa Junior}

Professor adjunto do Instituto de Psicologia da Universidade de Brasília

\section{* Endereço para correspondência:}

Fabrício Fernandes Almeida

SQS 315 Bloco "E" ap. 501 Asa Sul 70384-050 Brasília - DF - Brasil

Fax: (61) 3245-4378 - Tel.: (61) 8403-7886

E-mail: fabris.psicologia@gmail.com

Recebido 19/04/2007 Reformulado 13/02/2008 Aprovado 14/02/2008.

Arora, N. (2003). Interacting with cancer patients: The significance of physicians' communication behavior. Social Science and Medicine, 57, 791-806.

Azevedo, F. M., \& Thomas, C. V. (2002). Registro psicológico em prontuário. Revista da Sociedade Brasileira de Psicologia Hospitalar, 5(1/2), 26-28.

Belar, C. (1997). Clinical health psychology: A specialty for the 21st century. Health Psychology, 16, 411-416.

Conselho Federal de Psicologia. (2005). Código de ética profissional do psicólogo (XII Plenário do Conselho Federal de Psicologia). Brasília, DF: Autor.

Costa Junior, A. L. (2005). Análise de comportamentos de crianças expostas à punção venosa para quimioterapia. Tese de Doutoramento, Universidade de Brasília, Brasília, DF.

Costa Junior, A. L. (2005). Psicologia da saúde e desenvolvimento humano: o estudo do enfrentamento em crianças com câncer e expostas a procedimentos médicos invasivos. In M. A. Dessen \& A. L. Costa Junoir (Orgs.), A ciência do desenvolvimento humano: tendências atuais e perspectivas futuras (pp. 171-189). Porto Alegre: ArtMed.

Fabbro, L. (1999). Limitações jurídicas à autonomia do paciente. Bioética. Recuperado em 26 de outubro de 2006, do Portal Médico (CFM): http://www.portalmedico.org.br/revista/ bio1v7/limjuridicas.htm

Gioia-Martins, D. F., \& Rocha, A. J. (2001). Psicologia da saúde e o novo paradigma: novo paradigma? Trabalho apresentado no I Congresso de Psicologia Clínica, São Paulo, Brasil.

International Association for the Study of Pain Press. (1995). Core curriculum for professional education in pain (2nd ed. rev). Seattle: Author.

Kluck, M., \& Guimarães, J. R. (2002). Questões éticas e legais do prontuário do paciente: da teoria à prática. Trabalho apresentado no VIII CBIS - Congresso da Sociedade Brasileira de Informática em Saúde, Natal - RN.
Lopes, A. A. (1999). Prontuário orientado por problemas e evidências (POPE): modelo de prontuário médico para a assistência de pacientes internados e ambulatoriais. Desenvolvimento de material didático ou instrucional apostila. Recuperado em 6 de setembro de 2006, de: www. uniplac.br/revistamedica.pdf

Mararazzo, J. D. (1980). Behavioral health and behavioral medicine: Frontiers for a new health psychology. American Psychologist, 35, 807-817.

Massad, E., Marin, H. F., \& Azevedo Neto, R. (2003). O prontuário eletrônico do paciente na assistência, informação e conhecimento médico. São Paulo: USP/OPAS. Recuperado em 12 de agosto de 2006, de: http://www.med.fm.usp.br/dim/ livrosdim/prontuario.pdf

Nascimento, A. L., Andrade, M. G., Assed, A. M. P., \& Brasil, M. A. A. (2006). Um modelo para o parecer psiquiátrico no hospital geral. Jornal Brasileiro de Psiquiatria, 55(2), 102-107.

Poch, F. V., \& Caparrós, C. B. (2000). Afrontamiento del periodo de exámenes y sintomatología somática autoinformada en un grupo de estudiantes universitarios. Revista Electrónica de Psicologia, v. 4, n. 1, 2000. Recuperado em 25 de agosto de 2006, de: http://www.psiquiatria.com/psicologia/vol4num1/ art $5 . h$ htm

Sebastiani, R. W. (2003). Psicologia de la salud en Brasil: 50 años de historia. Suma Psicológica, 10(1), 25-42.

Seidl, E. M. F., \& Trócolli, B.T. (2006). Desenvolvimento de escala para avaliação do suporte social em HIV/aids. Psicologia: Teoria e Pesquisa, 22(3), 317-326.

Turra, V. (2006). Avaliação psicológica inicial de pacientes no contexto de internação cirúrgica. Brasília, DF. Manuscrito não publicado. 